\title{
Interval Cancers and Duty of Candour, a UK Perspective
}

\author{
Eleanor Cornford ${ }^{1} \cdot$ Nisha Sharma ${ }^{2,3}$
}

Published online: 18 March 2019

(C) The Author(s) 2019

\begin{abstract}
Purpose of Review Interval cancers are defined as a cancer presenting in the interval following a negative screen. Interval cancers are important because they reduce confidence in the screening programme and have relatively worse prognostic features than screen-detected cancers. This review will look at the rates of interval cancers in the UK Breast screening programme and other population-based breast screening programmes in Europe. It will discuss the latest UK classification and look at factors affecting interval cancer rates. It will examine the pathological features of interval cancers and their mortality impact. It will also discuss the introduction of Duty of Candour and its relevance and impact in breast screening.

Recent Findings It will report on the effect of newer technologies on interval cancer rates.

Summary Interval cancers are not unexpected in population-based screening programmes. They are known to have relatively worse prognosis than screen-detected cancers and therefore the accurate monitoring of interval cancers is important both for quality improvement and education. The impact of newer technologies on interval cancer rates is currently limited as the studies are ongoing.
\end{abstract}

Keywords Breast cancer $\cdot$ Interval cancers $\cdot$ Duty of candour $\cdot$ Breast density

\section{Introduction}

An interval breast cancer is a cancer that is diagnosed in between screening episodes after a woman has received a "normal" result. They are important because they reduce confidence in the screening programme and have relatively worse prognostic features than screen-detected cancers.

This review will look at the rates of interval cancers in the UK Breast screening programme and other population-based breast screening programmes in Europe. It will discuss the

This article is part of the Topical Collection on Breast Cancer Imaging and Screening

Eleanor Cornford

eleanor.cornford@nhs.net

Nisha Sharma

nisha.sharma2@nhs.net

1 Thirlestaine Breast Unit, Cobalt House, Gloucestershire Hospitals Foundation Trust, Thirlestaine Road, Cheltenham, Gloucestershire GL53 7AS, UK

2 Breast Unit, Level 1 Chancellor wing, Leeds Teaching Hospital NHS Trust, Beckett Street, Leeds, West Yorkshire LS9 7TF, UK

3 University of Leeds, Leeds LS2 9JT, UK latest UK classification and look at factors affecting interval cancer rates. It will examine the pathological features of interval cancers and their mortality impact.

It will also discuss the introduction of Duty of Candour and its relevance and impact in breast screening.

\section{Definition, Classification and Standards}

The majority of screening programmes specify an interval cancer as an invasive cancer but it is important to remember when comparing programmes that some programmes also include ductal carcinoma in situ (DCIS) [1]. In the UK, cases of DCIS are not included in the UK definition but will still undergo a review process.

Another problem with comparing rates between different programmes is the availability of up-to-date validated data. Certainly in the UK the most recent published national data refers to screening years 2006-2008 [2], although data is available more readily at regional level.

In the UK, guidance produced by Public health England in 2017 states three reasons to report and monitor interval cancers [3•] 
1. To monitor rates of interval cancers in conjunction with rates of screen-detected cancer to provide information on whole programme performance.

2. To support education and learning objectives for screening services and individuals. This helps improve the quality of the service provided to women.

3. So that women who have had an interval cancer can understand whether any abnormality was present on their previous films and if they wish to receive the information.

All of these are important. With regard to number 3, from the outset of the National Health Service Breast Screening Programme, women in the UK have always had the opportunity to discuss findings from review of their previous images with a clinician involved in their care, a process referred to as "Disclosure of Audit". More recently "Duty of Candour" has been introduced in the UK and this will be discussed more fully later in the review [4•].

The most recently available national data from the UK quotes the following statistics [2].

- Of 1,625,063 women screened in 2007/8

- Overall interval cancer rate in 3 years following screen $(50-64)=2.86 / 1000$

- Interval cancer rate in 2 years following screen $(50-64)=$ $1.61 / 1000$

- Overall interval cancer rate in 3 years following screen $(50-70)=2.95 / 1000$

- Interval cancer rate in 2 years following screen $(50-70)=$ $1.64 / 1000$

Tornberg et al. compared the interval cancer rates in six European countries with organised, population-based screening and found rates of interval breast cancers ranged from 8.4 to 21.3 per 10,000 screenings [5].

The following classification system for radiological review of interval cancers has been used in the UK since 2017 [3•]

1. Satisfactory-Normal or benign mammographic features

2. Satisfactory with learning points - Seen with hindsight, difficult to perceive, not obviously malignant

3. Unsatisfactory-Appearance is obviously malignant

This varies slightly from the previous classification where cat 3 was defined as-Appearance suspicious of malignancy [6].

The majority of interval cancers fall into category 1 (approximately $77 \%$ ), whilst category 2 accounts for approximately $16 \%$ and category 3 approximately $7 \%$ [2]. It is likely that with the updated classification system the percentage of category 3 interval cancers will decrease. This will therefore make it difficult to ascertain whether any decrease is due to true improvements in the screening programme or solely to the updated classification system.
The UK has quality standards for all aspects of the Breast Screening programme. The standards for interval cancer rates were updated in 2017 and are as follows:

$<12$ months - 0.65/1000, $12<24$ months - $1.4 / 1000,24$

$<36$ months - 1.65/1000 (compared with $<24$ months - 1.2 / $1000,24<36$ months - $1.4 / 1000$ in previous standards) [7].

The rates were increased to reflect the background incidence increase since 1995 and also split into three rates for each year to allow greater accuracy.

\section{Factors Affecting Interval Cancer Rates}

Rates are affected by ascertainment methods, screening round length, underlying background breast cancer incidence within a given population, and also risk factors. To achieve accurate data, robust systems need to be in place with good communication between cancer registries and both screening and symptomatic breast services. In a three yearly screening programme, the interval cancer rate goes up by approximately $75 \%$ in the third year which is why most countries in Europe have adopted two yearly programmes. In a three yearly screening programme, if round length is significantly less than 36 months, interval cancer rates would fall whilst the converse would be true if round length was significantly longer.

Risk factors for development of interval breast cancers are high breast density, age, current use of hormone replacement therapy, and family history of breast cancer [8-11]. High breast density increases risk in two ways. It has a masking effect but also it is an independent risk factor for breast cancer.

In addition to these factors, it has also been shown that there is an association between the detection and treatment of high grade DCIS and subsequent prevention of invasive interval cancers. Duffy et al. obtained aggregate data for DCIS diagnoses for women aged 50-64 years who were invited to and attended mammographic breast screening from April 2003 to March 2007 (from the National Health Service Breast Screening Programme). Patient-level data for interval cancer arising in the 36 months after each of these were also analysed. The average frequency of DCIS detected at screening was 1.60 per 1000 women screened. There was a significant negative association of screen-detected DCIS cases with the rate of invasive interval cancers. Ninety percent of units had a DCIS detection frequency within the range of 1.00 to 2.22 per 1000 women; in these units, for every three screen-detected cases of DCIS, there was one fewer invasive interval cancer in the next 3 years [12]. The challenge for radiologists is to recall intermediate/high grade DCIS and not overcall cases of low grade DCIS. 


\section{Radiological Features}

As discussed, earlier monitoring and surveillance of interval cancers is very important. In the UK, the NHSBSP makes recommendations regarding the review process. It states that a minimum of two reviewers should review the images, that the previous screening mammograms, with all prior images that were available at the time of screening, should be reviewed by the readers independently and that the diagnostic images should then be reviewed to confirm that any subtle or suspicious signs detected on the previous screening images match the site of the confirmed breast cancer on the diagnostic images [3•]. However, the exact method of review varies widely which may account for variation in reported interval cancer rates. The ideal review method would involve blinded review of cases interspersed with normal screening mammograms. In reality due to time and clinical commitments, many interval cancer reviews are informed which naturally introduces bias into the process with the reviewer more likely to classify a case as category 2 or 3 than if the process was blinded. The mammographic features most likely to be missed are asymmetric densities, ill-defined masses, subtle distortions, and calcification [13, 14].

\section{Pathological and Biological Features}

In terms of detection, some tumours particularly lobular may have subtle mammographic appearances or even be occult. In addition, small high grade cancers may appear as well-defined masses and be misinterpreted as benign. Studies looking at the pathology, biology, and prognosis of interval cancers have consistently found that they have poorer prognostic features compared with screendetected cancers. Consistent findings are larger size, higher grade, and node positivity [1].

There is also published work on biomarkers. Collett et al. found that a basal epithelial phenotype (express cytokeratin and P-cadherin) is more frequent in interval breast cancers compared with screen-detected tumours. Patients with interval cancers were more likely to be younger, have dense breasts, ER negative tumours, and $\mathrm{p} 53$ expression [15]. Data from other authors is mixed but the majority have found a higher proportion of triple negative tumours and over expression of HER 2 in interval cancers [16-19]. Interestingly, Holm et al. compared biological markers in interval cancers in non-dense breasts $(\leq 20 \%$ mammographic density) with interval cancers in dense breasts ( $>40.9 \%$ mammographic density) and found that the cancers in the low density breasts were phenotypically more aggressive [10].

Breast cancer-specific survival in interval cancer is worse than in screen-detected cancer. However, this difference may partly be explained by lead or length time bias, resulting in an artificial increase in survival time due to the early diagnosis in screen-detected cancer or the detection of slow-growing tumours [20-22].

\section{Effect of New Technologies on Interval Cancer Rates}

There has been a transition from screen film mammography (SFM) to full field digital mammography (FFDM) in most screening programmes. Published data on the effect on interval cancer rates has mainly been at unit or regional level, showing mixed data but certainly no increase in interval cancer rates $[23,24]$. Sankatsing et al. have recently published national data from the Dutch screening programme assessing the effect of the transition to FFDM. Data of 7.3 million screens in women aged 49-74, between 2004 and 2011, were linked to the Netherlands Cancer Registry to obtain data on interval cancers. They found that during the transition from SFM to FFDM, there was a significant rise in cancer detection rate and a stable interval cancer rate, leading to increased programme sensitivity. Although the recall rate increased, programme specificity remained high [25]. Hoff et al. looked at the mammographic features and found that in previous mammograms of cancers missed at FFDM there was a nonsignificant trend towards a higher $\%$ of asymmetric densities ( 27 vs $10 \%, P=0.007)$ and a lower $\%$ of calcifications (18 vs $34 \%, P=0.185)$ compared with those missed at SFM [24].

The use of computer aided detection (CAD) has not been shown to reduce recall rates [26]. Digital breast tomosynthesis has been introduced in many countries with most large-scale studies demonstrating improved cancer detection rates. However, unless there is a corresponding reduction in interval cancer rates, the implication is that there will be little impact on mortality. It is still early days for results from the major studies but there have been several recent publications. Houssami et al. examined interval breast cancers ascertained at 2year follow-up for women involved in the "Screening with tomosynthesis or standard mammography" (STORM) population-based trial. In the study group, the interval cancer rate was $1.23 / 1000$ screens $(95 \% \mathrm{CI}$ 0.56 to 2.34$)$. In concurrently screened women who attended the same screening services and received 2Dmammography, the interval cancer rate was $1.60 / 1000$ screens (95\% CI 1.14 to 2.17). They concluded that the interval breast cancer rate amongst screening participants in the STORM trial was marginally lower than estimates amongst 2D-screened women [27•]. Skaane et al. have also published interval cancer data from the results from the prospective population-based Oslo Tomosynthesis Screening Trial. They found that although there was an increase in cancer detection rate and increased specificity there was no change in interval cancer rates [28]. 


\section{Duty of Candour}

Up until 2015, breast patients were offered results of interval cancer review according to "Disclosure of Audit" guidance [29]. This was a document produced by the NHSBSP and covered communication regarding interval cancers. It covered suggested methodology for the review process with advice on when and how to communicate in an open way whilst still minimising distress to both patients and professionals. This had to change following the introduction of Duty of Candour (DOC).

DOC is a Care Quality Commission (CQC) regulation (number 20) published in April 2015 in response to the Francis report (this was a report into incidents at a NHS hospital trust) [30, 31]. The key points about DOC are that NHS organisations must act in an open and transparent way when things go wrong, they must tell users as soon as practicable about notifiable safety incidents and results of investigations and that they must apologise. This applies to all aspects of NHS care. There is evidence that open communication reduces risk of litigation.

Within the act a "notifiable safety incident" means "any unintended or unexpected incident that occurred in respect of a service user during the provision of a regulated activity that, in the reasonable opinion of a health care professional, could result in, or appears to have resulted in - the death of the service user, where the death relates directly to the incident rather than to the natural course of the service user's illness or underlying condition, or severe harm, moderate harm or prolonged psychological harm to the service user" [30]. It became apparent very soon after the act was introduced that this would have a major potential impact on the breast screening programme, staff morale and recruitment as there was the perception by some NHS Hospitals that any interval breast cancer constituted a notifiable safety incident! Collaborative work between Public Health England, the CQC and screening representatives resulted in the publication of a new document, the aim of which was to advise on best practice in NHS Screening programmes to ensure compliance with DOC regulations [4•]. It explains that screening tests are not $100 \%$ accurate so will have false negatives and false positives and these should not automatically trigger DOC. It emphasises that interval cancers are not "unexpected" and that the screening information leaflet provided to all breast screening patients should inform that screening is not $100 \%$ accurate. The NHSBSP now also provides a leaflet to give to women at time of cancer diagnosis if they are in the screening age group informing her that a review of any previous screening mammograms will take place. It is imperative to remember that patient choice is very important and that she may not wish to know results of this review. This is her right and DOC respects that. Clinicians also need to remember that timing of disclosure is very important and we cannot apply "As soon as reasonably practicable" as it may not be in her best interest. The review involves examining both the process and imaging and if it is felt that there was either a process failure or that interpretation fell below an expected standard then DOC regulations should apply. As part of the collaborative work, it was also felt necessary to produce new guidance on interval cancers to ensure a clearer and more consistent approach. As stated earlier, this resulted in Category 3 changing from Unsatisfactory-Appearance suspicious of malignancy [6] to Unsatisfactory-Appearance is obviously malignant [3•]. It was felt that this made clearer the link between classification and what action is warranted. It is still too early to assess what impact this legislation will have on the breast screening programme but certainly the PHE publications make it easier to ensure consistency across different units.

\section{Conclusions}

Interval cancer rates within the context of breast screening are important metrics. Interval cancers are known to have relatively worse prognosis than screen-detected cancers and therefore the accurate monitoring of interval cancers is important. This is particularly the case when new imaging technologies are recommended to replace $2 \mathrm{D}$ digital mammography as a screening tool. Tomosynthesis has been shown to be a promising screening tool but the data regarding the impact on interval cancer rates is limited as many studies are still ongoing.

\section{Compliance with Ethical Standards}

Conflict of Interest Eleanor Cornford and Nisha Sharma declare no conflicts of interest relevant to this manuscript.

Human and Animal Rights and Informed Consent This article does not contain any studies with human or animal subjects performed by any of the authors.

Open Access This article is distributed under the terms of the Creative Commons Attribution 4.0 International License (http:// creativecommons.org/licenses/by/4.0/), which permits unrestricted use, distribution, and reproduction in any medium, provided you give appropriate credit to the original author(s) and the source, provide a link to the Creative Commons license, and indicate if changes were made.

\section{References}

Papers of particular interest, published recently, have been highlighted as:

- Of importance

1. Houssami N, Hunter K. The epidemiology, radiology and biological characteristics of interval breast cancers in population mammography screening. NPJ Breast Cancer. 2017;3:12.

2. Michalopoulos D, Dibden A, Duffy SW. National collation of breast screening interval cancer data 2006-2008. published 2015.

3. Guidance on Interval cancers, Reporting, classification and monitoring of interval cancers and cancers following previous 
assessment 2017 PHE publications, gateway number: 2017216. This documents helps define how interval cancers are classified to ensure that there is standardisation within the UK. This is important regarding education but also auditing practise going forward.

4. Guidance on applying Duty of Candour and disclosing audit results, 2016. PHE publications gateway number: 2016343. This is an important document outlining the processes regarding Duty of Candour and Disclosure of audit. It emphasises the importance of being honest and objective when dealing with interval cancers.

5. Tornberg S, Kemetli L, Ascunce N, Hofvind S, Anttila A, Sèradour $\mathrm{B}$, et al. A pooled analysis of interval cancer rates in six European countries. Eur J Cancer Prev. 2010;19:87-93.

6. Quality Assurance Guidelines for Breast Cancer Screening Radiology NHSBSP Publication No 59, 2011.

7. NHS BSP Consolidated standards, 2017 PHE publications gateway number: 2016720

8. Boyd NF, Huszti E, Melnichouk O, Martin LJ, Hislop G, Chiarelli A, et al. Mammographic features associated with interval breast cancers in screening programs. Breast Cancer Res. 2014;16(4): 417. https://doi.org/10.1186/s13058-014-0417-7.

9. Wanders JOP, Holland K, Karssemeijer N, Peeters PHM, Veldhuis WB, Mann RM, et al. The effect of volumetric breast density on the risk of screen-detected and interval breast cancers: a cohort study. Breast Cancer Res. 2017;19:67.

10. Holm J, Humphreys K, Li J, Ploner A, Cheddad A, Eriksson M, et al. Risk factors and tumor characteristics of interval cancers by mammographic density. J Clin Oncol. 2015 Mar 20;33(9):1030-7. https://doi.org/10.1200/JCO.2014.58.9986.

11. Lowery JT, Byers T, Hokanson JE, Kittelson J, Lewin J, Risendal B, et al. Complementary approaches to assessing risk factors for interval breast cancer. Cancer Causes Control. 2011;22:23-31.

12. Duffy SW, Dibden A, Michalopoulos D, Offman J, Parmar D, Jerkins J, et al. Screen detection of ductal carcinoma in situ and subsequent incidence of invasive interval breast cancers: a retrospective population-based study. Lancet Oncol. 2016;17(1):10914. https://doi.org/10.1016/S1470-2045(15)00446-5.

13. Evans AJ, Kutt E, Record C, Waller M, Bobrow L, Moss S. Radiological and pathological findings of interval cancers in a multi-centre, randomized, controlled trial of mammographic screening in women from age 40-41 years. Clin Radiol. 2007;62(4):348-52.

14. Hofvind S, Geller B, Skaane P. Mammographic features and histopathological findings of interval breast cancers. Acta Radiol. 2008;49:975-81.

15. Collett K, Stefansson IM, Eide J, Braaten A, Wang H, Eide GE, et al. A basal epithelial phenotype is more frequent in interval breast cancers compared with screen detected tumours. Cancer Epidemiol Biomark Prev. 2005;14:1108-12.

16. Weber RJ, van Bommel RM, Louwman MW, Nederend J, Voogd $\mathrm{AC}$, Jansen $\mathrm{FH}$, et al. Characteristics and prognosis of interval cancers after biennial screen-film or full-field digital screening mammography. Breast Cancer Res Treat. 2016;158:471-83.

17. Meshkat B, Prichard RS, Al-Hilli Z, Bass GA, Quinn C, O'Doherty A, et al. A comparison of clinical-pathological characteristics between symptomatic and interval breast cancer. Breast. 2015;24: 278-82.

18. Domingo L, Salas D, Zubizarreta R, Baré M, Sarriugarte G, Barata $\mathrm{T}$, et al. Tumor phenotype and breast density in distinct categories of interval cancer: results of population-based mammography screening in Spain. Breast Cancer Res. 2014;16:R3.
19. Caldarella A, Puliti D, Crocetti E, Bianchi S, Vezzosi V, Apicella P, et al. Biological characteristics of interval cancers: a role for biomarkers in the breast cancer screening. J Cancer Res Clin Oncol. 2013;139:181-5.

20. van der Waal D, Verbeek ALM, Broeders MJM. Breast density and breast cancer-specific survival by detection mode. BMC Cancer. 2018; 18:386.

21. Allgood PC, Duffy SW, Kearins O, O'Sullivan E, Tappenden N, Wallis MG, et al. Explaining the difference in prognosis between screen-detected and symptomatic breast cancers. Br J Cancer. 2011;104:1680-5. https://doi.org/10.1038/bjc.2011.144.

22. Mook S, Van't Veer LJ, Rutgers EJ, Ravdin PM, van de Velde AO, van Leeuwen $\mathrm{FE}$, et al. Independent prognostic value of screen detection in invasive breast cancer. J Natl Cancer Inst. 2011;103: 585-97. https://doi.org/10.1093/jnci/djr043.

23. Nederend J, Duijm LE, Louwman MW, Coebergh JW, Roumen RM, Lohle PN, et al. Impact of the transition from screen-film to digital screening mammography on interval cancer characteristics and treatment - a population based study from the Netherlands. Eur $\mathrm{J}$ Cancer. 2014;50(1):31-9. https://doi.org/10.1016/j.ejca.2013.09.018.

24. Hoff SR, Abrahamsen AL, Samset JH, Vigeland E, Klepp O, Hofvind S. Breast cancer: missed interval and screening-detected cancer at full-field digital mammography and screen-film mammography - results from a retrospective review. Radiology. 2012;264(2):378-86. https://doi.org/10.1148/radiol.12112074.

25. Sankatsing VDV, Fracheboud J, de Munck L, Broeders MJM, van Ravesteyn NT, National Evaluation Team for Breast cancer screening, NETB, et al. Detection and interval cancer rates during the transition from screen-film to digital mammography in population-based screening. BMC Cancer. 2018;18:256. Published online 2018 Mar 5. https://doi.org/10.1186/s12885-0184122-2.

26. Lehman CD, Wellman RD, Buist DS, Kerlikowske K, Tosteson AN, Miglioretti DL. Breast cancer surveillance consortium diagnostic accuracy of digital screening mammography with and without computer-aided detection. JAMA Intern Med. 2015;175(11): 1828-37. https://doi.org/10.1001/jamainternmed.2015.5231.

27. Houssami N, Bernardi D, Caumo F, Brunelli S, Fantò C, Valentini $\mathrm{M}$, et al. Interval breast cancers in the 'screening with tomosynthesis or standard mammography' (STORM) populationbased trial. Breast. 2018;38:150-3 This paper is important because it suggests that interval cancer rates may be reduced with the introduction of DBT as a screening tool but recognises that more data is required and the studies are ongoing and therefore these results need to be interpreted with caution.

28. Skaane P, Sebuødegård S, Bandos AI, Gur D, Østerås BH, Gullien $\mathrm{R}$, et al. Performance of breast cancer screening using digital breast tomosynthesis: results from the prospective population-based Oslo Tomosynthesis Screening Trial. Breast Cancer Res Treat. 2018;169(3):489-96. https://doi.org/10.1007/s10549-018-4705-2.

29. Disclosure of Audit results in cancer screening advice on best practice, Cancer Screening Series No 3 April 2006, ISBN 184463031 5.

30. CQC Regulation 20: Duty of Candour 2015 Information for all providers: NHS bodies, adult social care, primary medical and dental care, and independent healthcare. www.cqc.org.uk/sites/default/files/ 20150327 duty_of_candour_guidance final.pdf. Accessed Nov 2018

31. Francis R. Report of the Mid Staffordshire NHS Foundation Trust Public Inquiry. London: The Stationery Office; 2013.

Publisher's Note Springer Nature remains neutral with regard to jurisdictional claims in published maps and institutional affiliations. 